\title{
Bioengineering Thermodynamics: An Engineering Science for Thermodynamics of Biosystems
}

\author{
Umberto Lucia \\ Dipartimento Energia, Politecnico di Torino, Corso Duca degli Abruzzi 24, 10129 Torino, Italy \\ E-mail: umberto.lucia@polito.it
}

Received 01 August 2015, Revised 07 November 2015, Accepted 07 November 2015

\begin{abstract}
Cells are open complex thermodynamic systems. Energy transformations, thermo-electro-chemical processes and transports occur across the cells membranes. Different thermo-electro-biochemical behaviours occur between health and disease states. Moreover, living systems waste heat, the result of the internal irreversibility. This heat is dissipated into the environment. But, this wasted heat represent a sort of information, which outflows from the cell toward its environment, completely accessible to any observer. Consequently, the analysis of irreversibility related to this wasted heat can represents a new approach to study the behaviour of the cells. So, this approach allows us to consider the living systems as black boxes and analyze only the inflows and outflows and their changes in relation to the modification of the environment. Therefore, information on the systems can be obtained by analyzing the changes in the cell heat wasted in relation to external perturbations. In this paper, a review of the recent results obtained by using this approach is proposed in order to highlight its thermodynamic fundamental: it could be the beginning of a new engineering science, the bioengineering thermodynamics. Some experimental evidences from literature are summarized and discussed. The approach proposed can allow us to explain them.
\end{abstract}

Keywords: Entropy generation; exergy; irreversibility; living systems; medicine and biochemistry thermodynamics; membrane transport.

\section{Introduction}

Cancer represents one of the most serious causes of death worldwide. Indeed, according to World Health Organization, in 2008 , it accounted around the $13 \%$ of all deaths $\left(7.6 \times 10^{6}\right.$ people). Statistical projection in 2030 highlights deaths from cancer to over $11 \times 10^{6}$ people $[1,2]$.

Mechanical phenomena appears fundamental in any biological process at organ, tissue, cell and biomolecular motors, with consequences also on genes, such as c-MET, TP53, etc.: many processes in cells are related to nanomechanical properties of their membranes [3]. As a consequence of the experimental results that external forces or changes in cell or tissue determine some developments, physiological changes, but also diseases, a new biomedical engineering approach, based on the mechanical engineering of the open systems and named mechanobiology, has been introduced in the analysis of the living systems with a great number of related results in biomedicine [4]. This science can be considered an interdisciplinary field between mechanical sciences and biological and medical ones. On the base of system biology, the engineering approach to cell behaviour can develop the analysis of the bio-molecular transmission of the external signals in relation to their ability to alter the structure, function, and dynamics of a cell system [4]. So, the mechanobiology fundamental result is represented by the explanation of the molecular mechanisms of mechano-transduction, by which cells sense and respond to biomechanical signals and convert them into biochemical signals [4]. Indeed, a great number of receptors, such as ECM molecules, transmembrane proteins, cytoskeleton, nuclei, and lipid bilayer, are sensitive to mechanical stimuli and react to them by inside-outside and outside-inside transduction for cell.

But, life is a nonselective process with a cooperative superposition of processes, interrelated one another, because the physiological stimuli are seldom dynamic, depending on the magnitude, frequency, and duration of external perturbations. Consequently, mechanobiology cannot support completely biologists and physicians in their analysis and studies. Indeed, as in engineering science, thermal, chemical and electromagnetic phenomena could be addresses in order to obtain a full approach to biosystems. To do so, thermodynamic analysis must be introduced in mechanobiology, and more in general in biomedical engineering, in order to obtain a new physical and engineering science for biosystems, useful to biologists and physicians: the bioengineering thermodynamics is growing.

Indeed, engineering thermodynamics is the science which studies both energy and its best use in relation to the available energy resources with particular regards to energy conversion, including power production, refrigeration and relationships among the properties of matter, including also living matter. Energy is a thermodynamic property of any system in relation to a reference state, which changes during any process, while its total amount remains constant in relation to the universe (the system and its environment). In cells, many processes such as replication, transcription and translation need to convert molecular binding energy, chemical bond hydrolysis and electromagnetic gradients into mechanical work, related to conformational changes and displacements [5]. The biomechanical analysis of DNA has pointed out the connections among forces, thermodynamics, 
nano-mechanical and electromagnetic behaviour of biological systems and kinetics [6,7]. So, we can argue that thermodynamics could be part of the mechanobiological approach in order to improve this science by analysing the biosystems also from a thermal point of view.

Indeed, the fundamental role of thermodynamics in biology can be pointed out in some fundamental results in literature. Wilson, et al. [8] developed a thermodynamic relationships in mitochondrial oxidative phosphorylation, while, Waldeck, et al. [9] developed a nonequilibrium thermodynamics model describing the action of completely coupled $\mathrm{Ca}^{2+}$-ATPase by analysing the variation of the coupling stoichiometries with the magnitude of the electrochemical gradients, as the ATPase hydrolyzes ATP. Jin \& Bethke [10] used a nonequilibrium thermodynamic approach in the context of the chemiosmotic model of proton translocation and energy conservation, obtaining that the rate at which electrons pass through the respiratory chain in mitochondria and respiring prokaryotic cells is described by the product of three terms, one describing electron donation, one acceptance, and a third, the thermodynamic drive. Their result is the first to account for both thermodynamic and kinetic controls on the respiration rate by highlighting the nonlinear dependence of flux on electrical potential gradient, its hyperbolic dependence on substrate concentration, and the inhibiting effects of reaction products. Moreover, Turina, et al. [11] developed a thermodynamic analysis of the proton transport-coupled ATP synthesis and hydrolysis. Demirel [12] developed a linear nonequilibrium thermodynamics approach by assuming that the biosystem is in the vicinity of global equilibrium, obtaining a nonisothermal reactiondiffusion systems control of the behaviour of many transport and rate processes in physical, chemical and biological systems, such as pattern formation and chemical pumps. $\mathrm{He}$ has highlighted that a flow occurs without or against its primary thermodynamic driving force, which may be a gradient of temperature or chemical potential or reaction affinity. The analytical result lead to unique definitions of cross-coefficients between a chemical reaction and heat and mass flows in terms of kinetic parameters, transport coefficients and degrees of coupling. The result consists in new parameters fundamental to describe some coupled reaction-transport systems. Chowdhury \& Chanda [13] have recently improved the thermodynamic approach on the electro-mechanical coupling in voltage-gated ion channels.

But, all these fundamental thermodynamic approaches analyse particular biochemical and transport processes, representing a bases to develop a more general approach such to consider all the processes involved in the interaction between the bio-system and its environment. So, starting from these fundamental results an improvement towards a more general approach must be developed. Indeed, the first law of thermodynamics expresses the conservation of energy, while the second law states that entropy continuously increases for the system and its environment [14,15] and introduces the statistical and informational meaning of global quantities, and the third law allows us to link the second law to quantum mechanics and thermodynamics [1619]. Consequently, thermodynamics could results the fundamental science for the analysis of open systems when energy transfers and energy transformations occur.

So, a powerful improvement can be developed in mechanobiology by introducing thermodynamics, with the consequence of suggesting a new science for the engineering analysis and control design of biosystems: a system theory which link together engineering thermodynamics, mechanobiology, biomedical engineering, biothermodynamics, bio-energy and systems biology.

In this paper, a review of the physical bases of this new approach is summarized and the first results are discussed. To do so, in Section 2 the thermodynamic fundamentals are summarized, in Section 3 the use of the engineering thermodynamic results in biosystems is proposed.

\section{The Thermodynamic Fundamentals}

Nature, from a physical, biological, chemical and mathematical point of view, is a complex system, while from an engineering point of view, it is the "first" engineer! But, for scientists and engineers, dynamics and evolution of complex systems are not easy to predict. A fundamental approach to study complex system is thermodynamics. Indeed, this science is brief, simple, unambiguous, and continuously improving, able to provide global (engineering and irreversible thermodynamics), local (statistical thermodynamics), linear (classical thermodynamics) and complex (non-equilibrium and non-linear thermodynamics) approaches to natural systems. But, the result of too many schools of thermodynamics is the difficult in communication between thermodynamicists and other scientists and, also, among themselves. The solution is to try to obtain a unified approach based on the fundamentals of physics.

Carnot [20] pointed out the existence of a well calculable limit for any conversion rate of the heat into the kinetic energy and work and Clausius [21] introduced the entropy in order to analyze just the dissipative processes [22]. But, the new thermodynamic standpoint for the evaluation of the lost exergy was introduced, independently, by Gouy [23-26], in 1889, and by Stodola [27], in 1905.

Recently, it has been highlighted that any effect in Nature is always the consequence of the dynamic balances of the interactions between the real systems and their environments [28-39]. Energy balances are the results of the exchange of energy, better exergy [41], between any real system and its environment [42-45]. The real systems evolution is always related to the decrease of their free energy, in the least time [16-19].

Just these noticeable considerations represent the fundamentals for a possible proposal for the unification of the too many schools of thermodynamics just starting from two fundamental concepts of physics: interactions and flows. The result is the analytical formulation of flow-based analysis in thermodynamics, which can play the role of a "rallying point" of all the schools of thermodynamics. Indeed, if we consider natural systems we can highlight that they are always open systems, which means that they can exchange heat and mass with their environment. So, the interaction with the environment is a fundamental concept for the thermodynamic analysis.

We consider the environment as a thermostat [46-48] and the system with its environment is an adiabatic closed system [49]. But, for an adiabatic close system, the total entropy always increases, as a consequence of the second law $[46,48,49]$. This total entropy can be written as $[50,51]$ :

$d S=d_{i} S+d_{e} S$

where $d S$ is the variation of the total entropy, $d_{e} S$ is the entropy variation for interaction between the open system considered and its environment, and $d_{i} S$ is the entropy variation due to irreversibility, such that: 
$\frac{d s}{d t} \geq 0$

Now, we can write Eq. (1) as [52]:

$\frac{d S}{d t}=\int_{V}\left[-\nabla \cdot\left(\frac{\mathbf{Q}}{T}\right)+\dot{s_{g}}\right] d V$

where $\mathrm{Q}$ is the heat flow, $T$ is the temperature, $V$ is the volume, $t$ is the time and $\dot{s}_{g}$ is the density of the entropy generation rate.

Now, we consider that the stationary states of the open system correspond to the equilibrium states of the adiabatic closed system. Considering the system together with its environment, we are analyzing an adiabatic closed system, so the entropy variation for the volume considered is maximum at the equilibrium $[47,48]$ :

$d S=0 \Rightarrow-\nabla \cdot\left(\frac{\mathrm{Q}}{T}\right)+\dot{s_{g}}=0$

and

$\nabla \cdot\left(\frac{\mathbf{Q}}{T}\right)=\dot{s_{g}}$

This last relation allows us to state that the flows between the system and its environment cause the entropy generation density. Now, considering that the rate of entropy generation density can be written as [52]:

$\dot{s_{g}}=\sum_{k} \mathbf{J}_{k} \cdot \mathbf{X}_{\mathbf{k}}$

where $\mathbf{J}_{k}$ is the flow of the $k$-th quantity involved in the process considered and $\mathbf{X}_{k}$ is the conjugated thermodynamic force [52]. In relation to the mathematical form (6) we must highlight the Curie principle [53] for which fluxes and forces of different tensor properties are never coupled in isotropic systems, so that we can consider the different component to be always independent one another [52].

Now, considering that:

$\nabla \cdot\left(\frac{\mathbf{Q}}{T}\right)=Q \cdot \nabla\left(\frac{1}{T}\right)+\frac{1}{T} \nabla \cdot \mathbf{Q}=\sum_{k} \mathbf{J}_{k} \cdot \mathbf{X}_{k}$

Eq. (5) becomes:

$\frac{1}{T} \nabla \cdot \mathbf{Q}=\sum_{k} \mathbf{J}_{k} \cdot \mathbf{X}_{k}-\mathbf{Q} \cdot \nabla\left(\frac{1}{T}\right)$

in agreement with Le Chatelier's principle [54], for which any change in concentration, temperature, volume, or pressure generates a readjustment of the system in opposition to the effects of the applied changes in order to establish a new equilibrium, or stationary state. It follows that the fundamental imperative of nature is to consume free energy in least time. This quest will yield the ubiquitous scale-free patterns [54]. Any readjustment of the state of the system can be obtained only by generating fluxes of free energy which entail any process where the system evolves from one state to another. The free energy "fuels" evolutionary processes so that the basic building blocks of the nature, the quanta of energy, are either absorbed from the surroundings to the systems in the form bound energy or emitted from the systems to the surroundings as freely propagating photons.

\section{From Engineering Open Systems to Cells}

The approach previously summarized allows us to introduce some considerations [55-59]:
1. The energy lost by a system is gained by the environment, consequently, the information lost by the system is gained by the environment: here the problem is to codify this information;

2. The environment is completely accessible by any observer, so it is easy to collect data on the lost energy of any system;

3. The flows cause entropy generation variations, consequently we can evaluate the entropy generation to obtain information to the flows, even when we are unable to evaluate the flows themselves;

4. The entropy generation is a global quantity, so we can obtain global information on the cells, but from a biomedical point of view just the global cells behavior is the useful information.

By taking into account these physical considerations [14, 28-39, 43-46] a completely new approach to complex systems has been developed: the aim is to obtain an approach related to the spontaneous behaviour of the natural systems, based on the following considerations:

1. An open irreversible real linear or non-linear system is considered;

2. Each process has a finite lifetime $\tau$;

3. What happens in each instant in the range $[0, \tau]$ is unknown, but what has happened after the time $\tau$ is always known;

4. The local equilibrium is required to define the global thermodynamic quantities;

5. The balance equation is a balance of exergy fluxes.

The fundamental hypotheses of this approach are:

1. The work lost for irreversibility is energy collected by the environment;

2. The environment temperature, always considered constant during any process.

This approach is interesting in science and engineering because it allows us to analyse complex systems, partially inaccessible, on which our knowledge is, consequently, only partial. Biological systems represent example of such systems because they are able to adapt to the variation of environmental conditions; indeed, cells attain their "optimal" performance by a selection process driven by their environment. The resultant effect is a redistribution of energy and mass flows in their metabolic network, by using regulatory proteins.

The bioengineering thermodynamic approach to biological systems consists in the analysis of the biological optimization process realized by the nature. It is no more than the classical and engineering thermodynamic analysis of the steady-state flux distribution [55-59], which are no more than the metabolic flows. So, in the analysis of the metabolic networks, consequently to the results of a sufficiently large set of experiments, the entropy production rate of the cells has been suggested to be maximized at the optimized state [55-59].

Entropy was introduced in classical thermodynamics in relation to the equilibrium states and reversible transformations. It is a state function. When an irreversible system is considered [46, 49, 60-63], mathematical inequalities appear, while physician and engineers need to use equality. So, entropy generation was introduced just to avoid these inequalities. So, the second law defines the entropy variation, $\Delta S$, for an irreversible system, as $[14,28$, $31,38,58]$ : 
$\Delta S=\int\left(\frac{\delta Q}{T}\right)_{r e v}=\Delta S_{e}+S_{g}$

where $\Delta S_{e}$ is the entropy variation that could be obtained through a reversible path on which the system exchanges the same fluxes across its boundaries, $S_{g}$ is the entropy generation, i.e. the entropy variation due to irreversibility. But, during any process, only entropy really varies, so nothing is really produced or generated [64]. Consequently, entropy generation, the entropy variation due to irreversibility, $S_{g}$, is not more than a quantity that allows us to measure how far the system is from the state that the system could attain through a reversible path [36]. The analytical definition of entropy generation is defined as [49]:

$S_{g}=\int_{0}^{\tau} \dot{S}_{g} d t$

where $\tau$ is the lifetime of the process under consideration, which can be defined as the range of time in which the process occurs [49] and:

$\dot{S}_{g}=\frac{d S}{d t}-\sum_{i=1}^{n} \frac{\dot{Q}}{T_{i}}-\sum_{\text {in }} G_{i n} S_{\text {in }}+\sum_{\text {out }} G_{\text {out }} S_{\text {out }}$

where $Q$ is the heat exchanged, $T$ is the temperature of the thermal source, $s$ is the specific entropy and $G$ is the mass flow. Moreover, for any open system, the entropy balance in a local form can be written as [52]:

$\frac{\partial s}{\partial t}+v \nabla \cdot\left[\frac{\mathbf{Q}}{T}+\sum_{i} \rho_{i} s_{i}\left(\dot{x}_{i}-\dot{x}_{B}\right)\right]=v \sigma$

where $s=S / m$, is the specific entropy, $S$ is the entropy, $\sigma$ is the entropy production density, $v$ is the specific volume, $\mathbf{Q}$ is the heat flow, $\dot{\mathbf{x}}_{i}$ is the relative velocity in relation to the centre of mass reference, and $\dot{\mathbf{x}}_{B}$ is the centre of mass velocity. Now, we consider the Gibb's relation can be introduced [52]:

$T \frac{d s}{d t}=\frac{d u}{d t}+p \frac{d v}{d t}-\sum_{i} \mu_{i} \frac{d c_{i}}{d t}$

where $s$ is the specific entropy, $u$ is the internal specific energy, $v$ is the specific volume, $p$ is the pressure, $\mu_{i}$ are the chemical potentials, $c_{i}$ is the concentrations, $T$ is the temperature and $d / d t=\partial / \partial t+\dot{\mathbf{x}}_{B} \cdot \nabla$. We consider also the following relations for the specific internal energy [52]:

$$
\begin{aligned}
& \frac{d u}{d t}=\frac{d q}{d t}-p \frac{d v}{d t}-v \mathbf{\Pi}: \nabla \dot{\mathbf{x}}_{B}+v \sum_{k} \mathbf{J}_{k} \cdot \mathbf{F}_{k} \\
& \frac{d q}{d t}=-v \nabla \cdot \mathbf{Q} \\
& \frac{d c_{i}}{d t}=-\left(\nabla \cdot \mathbf{J}_{i}-\sum_{j} v_{i j} J_{j}\right)
\end{aligned}
$$

where $q$ is the heat per unit mass, $\Pi=\mathbf{P}-p \mathbf{I}$ with $\Pi$ total pressure tensor, $p$ hydrostatic pressure and $\mathbf{I}$ identity matrix of which the elements are $I_{j k}=\delta_{j k}=1$ if $j=k$ and 0 in the other cases, $\mathbf{a}: \mathbf{b}=\sum_{i j} a_{i j} b_{j i}$ is the product between two tensors $\mathbf{a}$ and $\mathbf{b}, \mathbf{J}_{k}=\rho_{k}\left(\dot{\mathbf{x}}_{i}-\dot{\mathbf{x}}_{B}\right)$ is the diffusion flows and $\mathbf{F}_{k}$ are the forces, $J_{j}$ is the chemical reaction rate of the $j$-th chemical reaction and $v_{i j}$ are quantities such that if they are divided by the molecular mass of the $i$-th component they are proportional to the stoichiometric coefficients. So, by introducing the relations (14), (15) and (16) in the relation (13), considering the electro-chemical affinity $\tilde{A}=\mathcal{A}+Z \Delta \phi$ related also to $\mathrm{pH}$ variation and the electric field variation, with $\mathcal{A}_{j}=\Sigma_{k} v_{k j} \mu_{j}, Z$ the electric charge per unit mass, $\phi$ the electrostatic potential, and $d / d t=\partial / \partial t+\dot{\mathbf{x}}_{B} \cdot \nabla$, the relation (12) holds [52]:

$$
\begin{aligned}
& \dot{s}=-\frac{v}{T^{2}} \mathbf{Q} \cdot \nabla T-v \sum_{k} \mathbf{J}_{k} \cdot \nabla\left(\frac{\widetilde{\mu}_{k}}{T}\right)-\frac{v}{T} \boldsymbol{\Pi}: \nabla \dot{\mathbf{x}}_{B}- \\
& \frac{v}{T} \sum_{j} J_{j} \tilde{\mathcal{A}}_{j}+\frac{v}{T} \sum_{k} \mathbf{J}_{k} \cdot \mathbf{F}_{k}
\end{aligned}
$$

From which a general form for the entropy generation can be obtained:

$$
\begin{aligned}
& S_{g}=\int_{V} d V\left[-\int_{0}^{\tau_{1}} \frac{v}{T^{2}} \mathbf{Q} \cdot \nabla T d t-\int_{0}^{\tau_{2}} v \sum_{k} \mathbf{J}_{k} \cdot \nabla\left(\frac{\widetilde{\mu}_{k}}{T}\right) d t-\right. \\
& \left.\int_{0}^{\tau_{3}} \frac{v}{T} \boldsymbol{\Pi}: \nabla \dot{\mathbf{x}}_{B} d t-\int_{0}^{\tau_{4}} \frac{v}{T} \sum_{j} J_{j} \tilde{A}_{j} d t+\int_{0}^{\tau_{5}} \frac{v}{T} \sum_{k} \mathbf{J}_{k} \cdot \mathbf{F}_{k} d t\right]
\end{aligned}
$$

where:

1. $S_{g, f f}$ is the entropy generation due to the thermal flux driven by temperature difference;

$S_{g, d c}$ is the entropy generation due to the diffusion current driven by chemical potential gradients, with $\tilde{\mu}=$ $\mu+Z \phi$ electrochemical potential, $\mu$ chemical potential;

2. $S_{g, v g}$ is the entropy generation due to the velocity gradient coupled with viscous stress;

3. $S_{g, c r}$ is the entropy generation due to the chemical reaction rate driven by affinity, always positive;

4. $S_{g, d e}$ is the entropy generation due to the dissipation due to work by interaction with the environment;

and $\tau_{i}, i \in$ are the lifetimes [65] of any process.

Cells are biological engines with specifically ordered chemical reactions. A part of their energy is lost as heat outflow and only the resulting products of biochemical processes are known, while any individual step is largely inaccessible. Consequently, the study of cells can be developed by introducing the black box model where only the spontaneous cell flows are considered. Any possible control of the cell behaviour is introduced by a control of the flows themselves: it is related to the entropy generation $S_{g, d e}$ due to work lost by interaction with external forces and fields (electric, magnetic, mechanical, etc.).

Therefore, the spontaneous exchange of heat represents the interaction or, here, the spontaneous communication between the cell and its environment [66-69], i.e., the heat flow across the cells membrane. In particular, considering that [50]:

$\dot{S}_{g}=\mathbf{J}_{\mathbf{U}} \cdot \nabla\left(\frac{1}{\mathrm{~T}}\right)+\sum_{\mathrm{i}} \mathbf{J}_{N_{i}} \cdot \nabla\left(\frac{\widetilde{\mu}}{\mathrm{T}}\right)$

where $\mathbf{J}_{U}$ is the energy flow, $T$ is the temperature, $\tilde{\mu}=\mu+$ $Z \phi$ is the electrochemical potential, $\mu$ is the chemical potential, $Z$ is the electric charge per unit mass, $\phi$ is the electrostatic potential and $\mathbf{J}_{\mathrm{Ni}}$ is the molar flow (ions included). This heat flow is the consequence of irreversible processes within cells and this is easily developed by using the approach previously introduced and named bioengineering thermodynamics. Indeed, it is easily to highlight that any irreversible process inside the cell causes flows across the cell membrane and that, conversely, any flow across the membrane causes a variation of the entropy generation [70-74]. This entropy generation is related to the work (more general energy) lost for irreversibility and to the temperature of the system's environment, which is considered as a thermostat. This approach considers the total entropy generation as the fundamental quantity useful to this work lost. So, this quantity has been evaluated. The full 
approach used to evaluate any term of the entropy generation is developed in Refs. [72], while, in this paper, the result is summarized as follows:

1. The volume of the cell is evaluated by a characteristic length, in transport phenomena usually considered the diameter of the cell approximated as the diameter of a sphere:

$L=\left(\frac{6 V}{\pi}\right)^{1 / 3}=2 r$

with $r$ being the cell radius;

2. The mean environmental temperature can be assumed as $T_{0}=310 \mathrm{~K}$ and the mean cell temperature has been estimated to be $T_{0}+\Delta T$. The quantity $\Delta T$ would be experimentally evaluated for different cells lines in relation to their metabolism;

3. The internal energy density results in $u=3.95 \times 10^{7} \mathrm{Jm}^{-}$ ${ }^{3}$, being calculated as the ratio between the ATP energy, $U=$ $3 \times 10^{-7} \mathrm{~J}$ and the mean value of the cell inside the human body, $V=7600 \mu \mathrm{m}^{3}$. It must be emphasized that this is an approximation because the cell volume inside the human body is in the range of $200-15000 \mu \mathrm{m}^{3}$;

4. The thermal molecular mean velocity inside the cytoplasm is considered to be $\dot{x}_{t h}=5 \times 10^{-5} \mathrm{~ms}^{-1}$;

5. the membrane volume is calculated with

$V_{m}=\frac{4}{3} \pi r^{3}-\frac{4}{3} \pi\left(r-d_{e}\right)^{3}=0.992 \mathrm{~V}$

being $d_{e}=0.2 r$;

6. The chemical potential gradient can be approximated through the ratio between the mean value of the chemical potential $\mu=1.20 \times 10^{-9} \mathrm{~J} \mathrm{~kg}^{-1}$ and the membrane length $d_{m}=$ $0.01 \mu \mathrm{m}$, with the mean density being $\rho=1000 \mathrm{~kg} \mathrm{~m}^{-3}$;

7. The viscosity is taken to be $6.91 \times 10^{-3} \mathrm{~N} \mathrm{~s} \mathrm{~m}^{-2}$;

8. $\eta \sim 2.07 \times 10^{-3} \mathrm{~N} \mathrm{~s} \mathrm{~m}^{-2}$ at $30^{\circ} \mathrm{C}$;

9. $\dot{x}_{B}$ is set as $3.0 \times 10^{-6} \mathrm{~m} \mathrm{~s}^{-1}$;

10. $\tau_{1}$ is the time related to the thermal flow driven by temperature difference. It can be assessed considering that the time constant of the thermal transient for heat conduction is:

$\tau_{c v} \approx \frac{\rho c V}{h A}$

with $\rho \approx 1000 \mathrm{~kg} \mathrm{~m}^{-3}$ density, $V$ the cell volume, $A$ the external cell surface, $c \approx 4186 \mathrm{~J} \mathrm{~kg}^{-1} \mathrm{~K}^{-1}$ specific heath, and $h$ the convection heat transfer coefficient evaluated as:

$h \approx 0.023 \frac{\lambda}{L} \operatorname{Re}^{0.8} \operatorname{Pr}^{0.35}$

where $\lambda \approx 0.6 \mathrm{~W} \mathrm{~m}^{-1} \mathrm{~K}^{-1}$ of heat conductibility [111], $L$ the characteristic dimension of the cell (here we have considered the diameter), $\operatorname{Re} \approx 0.2$ the Reynolds number and $\operatorname{Pr} \approx 7$ the Prandtl number. The process would have occurred in a time $\tau_{1} \approx 5 \tau_{c v}$. For human cells this value can be considered in the range $15-269 \mathrm{~ms}$;

11. $\tau_{2}$ is the time related to the diffusion current driven by chemical potential gradients. It can be evaluated as:

$\tau_{2} \approx \frac{d}{D}$

with $d=0.01 \mu \mathrm{m}$, i.e., the length of the membrane, and $D$ being the diffusion coefficient. Considering that the diffusion coefficient of glucose is approximately $10^{-9} \mathrm{~m}^{2} \mathrm{~s}^{-1}$ it follows that $\tau_{2} \approx 10 \mathrm{~s}$;

12. $\tau_{3}$ is the time related to the velocity gradient coupled with viscous stress. This time can be evaluated as the propagating time of a mechanical wave on the surface of the cell:

$\tau_{3} \approx \frac{2 \pi r}{c}$

with $c \sim 1540 \mathrm{~m} \mathrm{~s}^{-1}$ the sound velocity, considered to be the same in biological tissue;

13. $\tau_{4}$ is the time related to the chemical reaction rate driven by affinity and it can be evaluated considering the magnitude order of a chemical reaction in a cell $\left(\sim 10^{-7}\right.$ mol s$\left.{ }^{-1} \mathrm{l}^{-1}\right)$. Moreover, we consider that the moles number is proportional to the density of the chemical species (for glucose $1540 \mathrm{~kg} \mathrm{~m}^{-3}$ ) and the volume of the cell itself. It follows that this time is in the range $17-1283 \mathrm{~ns}$;

14. $\tau_{5}$ is the time related to the dissipation due to work by interaction with the external forces. It depends on the interaction considered

15. $S_{g, t f} \approx \frac{u L^{2} \dot{x}_{t h}}{6 T^{2}} \Delta T \tau_{1}=\alpha \cdot \Delta T \cdot L^{2}$ is the entropy generation due to the thermal flux driven by temperature difference;

16. $S_{g, d g} \approx \frac{\dot{x}_{t h} V_{m}}{T} \frac{\sum_{i} \rho_{i}\left(\mu_{i, o s}-\mu_{i, i s}\right)}{d_{m}} \tau=\beta \cdot L^{3}$ is the entropy generation due to the diffusion current driven by chemical potential gradients;

17. $S_{g, v g} \approx \frac{4 \pi}{T} \eta \frac{\dot{x}_{B}^{2}}{r d_{e}} \tau_{3}=\frac{\gamma}{L^{2}}$ is the entropy generation due to the velocity gradient coupled with viscous stress;

18. $S_{g, c r} \approx V \tau_{4} \sum_{i} \frac{N_{i}}{T} \tilde{A}_{i}=\varepsilon L^{3}$ is the entropy generation due to the electrochemical reaction rate driven by electrochemical affinity $\tilde{\mathcal{A}}=\mathcal{A}+Z \Delta \phi$, with $\mathcal{A}$ chemical affinity, related also to $\mathrm{pH}$ variation and the membrane's electric field variation;

19. $S_{g, d e} \approx-\int_{V} d V \int_{0}^{\tau_{5}} \frac{v}{T} \sum_{k} \mathbf{J}_{k} \cdot \mathbf{F}_{k} d t=-\kappa L^{3} \quad$ is the entropy generation due to dissipation caused by work through interaction with the environment; it depends on the kind of field considered;

20. $\alpha, \beta, \gamma, \varepsilon, \kappa$ are coefficients depending on the environmental parameters (temperature, chemicals concentrations, etc.) and on the cell characteristics (density, volume, etc.)

21. $L$ is a characteristic length, introduced as usually done in transport phenomena.

Consequently, the total entropy generation for a cell can be written as a function of the mean diameter:

$S_{g} \approx \alpha \cdot \Delta T \cdot L^{2}+(\beta-\kappa) L^{3}+\frac{\gamma}{L^{2}}+\varepsilon L$

Many experiments have been developed to obtain nanomechanical properties of cell membranes, and the bioengineering thermodynamics has been verified by considering them. Here, just one of them is summarized, the experimental results of Pelling et al. [3] on the elastic response of the Saccharomyces cerevisiae membrane to an external applied force. To compare the theoretical results with the experimental ones, the power lost due to the irreversibility for elastic deformation has been evaluated by the following relations: 
$\dot{W}_{\lambda}=\frac{T S_{g, v g}}{\tau_{3}} \frac{\Delta V}{V}=\frac{1}{2} \varrho A^{2}(2 \pi v)^{2} c$

where $\Delta V$ is the variation of the volume due to elastic deformation and the original volume and $V$ is the original volume, so the greater is the cell the less will be the effect of the nanomechanical stress, $A=(3.0 \pm 0.5) \mathrm{nm}$ is the amplitude of the elastic perturbation, $\rho$ is the density of the medium, $v=1.6 \mathrm{kHz}$ is the frequency of the elastic wave and $c \sim 1540 \mathrm{~m} \mathrm{~s}^{-1}$ is the sound velocity in the medium considered. So, it results $(1.11 \pm 0.37) \times 10^{-4} \mathrm{~W}$. The value obtained by using the $(1.18 \pm 0.04) \times 10^{-4} \mathrm{~W}$. These two results agree by $94 \%$ and the theoretical value is inside the range of the experimental one.

Consequently, cell membrane changes its behaviour in relation to the ionic flows across its surface with effects on the chemical reactions inside the cell itself.

Moreover, an experiment has been developed to obtain also a direct proof. Therefore the spontaneous heat exchanged by the cell represents the interaction or the spontaneous communication between the cell and its environment. The proposed thermodynamic theory predicts that the temperature difference between cells with distinct metabolic characteristics can be amplified by an altered interaction with the external environment, due to the entropy generation term related to the interaction of the system with the external fields. The experiments carried out on cells exposed to low frequency electromagnetic waves consolidate the thermodynamic approach. Indeed, through infrared thermography a nondimensional number, thermal dispersion index, was evaluated. This number represents the inability of the cells to fit their thermal power to environmental changes. Primary fibroblasts display a high dispersion index, with a maximal value of $800 \%$ vs NIH3T3 immortalized line, which means that the primary fibroblasts adjust more efficiently their thermal production or dissipation than the NIH3T3 cells. This significant difference implies that, when exposed to selected environmental conditions, transformed cells dissipate heat more slowly than their normal counterpart. The results of this experimental approach demonstrate that selecting environmental conditions it is possible to appreciate distinct cellular phenotypes; these differences can be evaluated by thermal dispersion patterns measured by infrared thermography. The experiment confirmed the bioengineering thermodynamics theoretical results.

But, in literature, there exist other experiments which represent a confirmation on one hand, and which can be find an explanation on the theory itself. Indeed, they highlight the biophysical and biochemical consequences of the interaction between the cells systems and tissues and their environment, as expected by the previous discussion. Some of these experimental evidences can be summarized as follows:

1. in vitro studies:

- HeLa (human cervical cancer) and PC-12 (rat pheochromocytoma) cells, continuously exposed for $72 \mathrm{~h}$, to ELF-EMF of $(1.2 \pm 0.1) \mathrm{mT}$, at $60 \mathrm{~Hz}$, decrease in proliferation $18.4 \%(\mathrm{HeLa})$ and $12.9 \%(\mathrm{PC}-12)$ [75];

- HeLa cells exposed to PEMF of $0.18 \mathrm{~T}$, at $0.8 \mathrm{~Hz}$, for $16 \mathrm{~h}$ : they decrease of $15 \%$ in proliferation, as observed 24h later [76];

- $\quad$ PC-12 cells exposed to ELF-EMF at $50 \mathrm{~Hz}$ of different intensities and durations: transient decrease of the proliferation rate and morpholigical differentiation has been observed [77];

- Human colon adenocarcinoma cells exposed to ELFEMF of $1.5 \mathrm{mT}$ peak and $1 \mathrm{~Hz}$, for $360 \mathrm{~min}$ : they decrease in cell growth [78];

- HCA-2/1cch (human colon adenocarcinoma) cells exposed to $25 \mathrm{~Hz}, 1.5 \mathrm{mT}$, for $2 \mathrm{~h}$ and $45 \mathrm{~min}$ in presence of dexamethasone: they decrease of (55.84 \pm $7.35) \%$ in the relative cells number [79];

- HTB 63 (melanoma), HTB 77 IP3 (ovarian carcinoma), and CCL 86 (lymphoma; Raji cells) cell lin: $64 \mathrm{~h}$ exposure under a 7T uniform static magnetic field leads to reduction of viable cell number by $(19.04 \pm 7.32) \%$ (HTB 63), (22.06 \pm 6.19$) \%$ (HTB 77 IP3), and (40.68 $\pm 8.31) \%$ (CCL 86) [80];

- WiDr (human colon adenocarcinoma), MCF-7 (human breast adenocarcinoma), and MRC-5 (embryonal lung fibroblast) have been exposed to $3 \mathrm{mT}$ static $\mathrm{MF}$, modulated in amplitude with $3 \mathrm{mT}$ ELF-MF, at $50 \mathrm{~Hz}$, with a superimposition of ELF magnetic field, for 20 min. Both WiDr and MCF-7 cells showed morphological evidence of increased apoptosis. MRC5 cells remained intact and did not show any increase in apoptosis [81];

- HL-60 and ML-1 (Human Myeloblastic Leukemia) cells undergo apoptosis (detected through ladder typeDNA fragmentation) after exposure at $50 \mathrm{~Hz}, 45 \mathrm{mT}$ ELF-EMF for time periods of 1 and $2.5 \mathrm{~h}$ [82];

- SCL II (human squamous cell carcinoma) cells and AFC (human amniotic fluid) continuously exposed at $50 \mathrm{~Hz}, 0.8-1.0 \mathrm{mT} \mathrm{EMF}$, for $48 \mathrm{~h}$ and $72 \mathrm{~h}$ : they increase in the frequency of micronucleus (MN) formation and the induction of apoptosis has been observed [83];

- SH-SY5Y (human neuroblastoma) cells continuously exposed to a $900 \mathrm{MHz}$ radiofrequency radiation (SAR: $1 \mathrm{~W} \mathrm{\textrm {kg } ^ { - 1 }}$ ), for $24 \mathrm{~h}$ : reduction in the viability of neuroblastoma cells [84];

2. in vivo studies:

- Antitumor and immunomodulatory effects of pulsed magnetic fields have been observed when the pulse width is $238 \mu \mathrm{s}$, the peak magnetic field is $0.25 \mathrm{~T}$, the frequency $25 \mathrm{~Hz}, 1000 \mathrm{~Hz}$ and the magnetically induced eddy currents in B16-BL6 melanoma model mice is $0.79-1.54 \mathrm{~A} \mathrm{~m}^{-2}$. Exposure of mice in pulsed magnetic fields lasted in 16 days. Anticancer and immunomodulatory properties of pulsed magnetic stimulation and decrease of tumor growth and elevated production of tumor necrosis factor (TNF-a) have been observed in mouse spleens [85];

- Extremely low-frequency pulsed-gradient magnetic field with the maximum intensity of $0.6-2.0 \mathrm{~T}$, gradient of $10-100 \mathrm{~T} \mathrm{~m}^{-1}$, pulse width of $20-200 \mathrm{~ms}$ and frequency of $0.16-1.34 \mathrm{~Hz}$, presented antitumor and antiangiogenic properties, in exposed Kunming mice bearing murine tumor [86-88];

- Male Fischer-344 rats, subjected to the modified resistant hepatocyte model, were exposed to $4.5 \mathrm{mT}$ $120 \mathrm{~Hz}$ ELF-EMF. The results showed a decrease of more than $50 \%$ of the number and the area of $\gamma$ glutamyl transpeptidase-positive preneoplastic lesions, glutathione S-transferase placental expression, a decrease of proliferating cell nuclear antigen, Ki-67, and cyclin D1 expression. These results showed 
inhibition of preneoplastic lesions, through antiproliferative activity of ELF-EMF [89];

- Nude mice, bearing a subcutaneous human breast tumour (MDA-MB-435), were exposed for $70 \mathrm{~min}$ daily, for six consecutive weeks, to modulated MF (static with a superimposition of extremely low frequency fields at $50 \mathrm{~Hz}$ ), of total intensity of $5.5 \mathrm{mT}$. The anticancer activity of MF was compared to that of cyclophosphamide. The inhibition on spread and growth of lung metastases caused by MF was greater than that caused by cyclophosphamide [90];

3. clinic studies

- A combination of tumor-specific frequencies may have a therapeutic effect. A total of 1524 frequencies, ranging from 0.1 to $114 \mathrm{kHz}$, were identified from 163 cancer patients, while a compassionate treatment was offered to 28 patients with advanced cancer (breast, ovarian pancreas, colon, prostate, sarcoma and other types of cancer). The patients received a total of 278.4 months of experimental treatment and the median treatment duration was 4.1 months per patient. None of the patients, who received experimental therapy, reported any side effects of significance. Two of the patients presented a complete and partial response to the treatment and four patients presented stable disease. A woman, with breast cancer, showed a complete disappearance of some lesions, according to PET-CT (Positron emission tomography - computed tomography), and significant improvement of the overall condition. Thus, the tumor-specific frequencies provide an effective and well tolerated treatment which may present antitumor properties in end-stage patients [91];

- Eleven patients with mean age of 60 years and with stage IV, locally advanced or metastatic disease (adenocarcinoma, duct carcinoma, squamous cell carcinoma and other types), were enrolled in a human pilot study conducted. Patients were exposed for 5 days/week, over 4 weeks, according to two different static magnetic fields schedules: $20 \mathrm{~min}$ daily (4 patients) and 70 min daily (7 patients). Results showed that MF-exposed patients present mild or no side effects. Moreover, this pilot study supports the evidence that human exposure to MF with specific physical characteristics is associated with a favorable safety profile and good tolerability [92];

- Ten patients with recurrent glioblastoma was treated by using a 100-300 kHz magnetic fields. No serious adverse events were observed in all patients, after $>70$ months of cumulative treatment. The median time of disease progression and median overall survival were more than double than the reported medians of historical control patients. This type of fields can be used as a safe and effective treatment for cancer patients [93];

- In 2008, a pilot study was developed to investigate the safety and efficacy of low-intensity, intermediatefrequency electric fields in 6 patients (heavily pretreated with several lines of therapy) with metastatic solid tumors, while no additional standard treatment option was available to them. A device was used to emit the frequencies $100-200 \mathrm{kHz}$, at a field intensity of 0.7 $\mathrm{V} / \mathrm{cm}$. A patient presented $51 \%$ reduction in tumor size, after 4 weeks of fields' treatment. Also, an arrest of tumor growth was seen in three patients, during treatment. Despite the small number of patients, this study revealed that this type of electric fields presented lack of toxicity and significant efficacy in patients' treatment [94];

- In a recent phase I/II clinical study, 41 patients with advanced hepatocellular carcinoma (HCC), were subjected to very low levels of electromagnetic fields modulated at HCC specific frequencies $(410.2 \mathrm{~Hz}-$ 20365.3Hz). Patients were being administered with three-daily 60 min outpatient treatments, till the disease progression or death. During treatment no NCI grade 2, 3 or 4 toxicities (grades based on National Cancer Institute Common Terminology Criteria (CTC) for adverse events), were observed, while most of the patients reported complete disappearance or decrease of pain shortly after treatment initiation. Four patients presented a partial response to the treatment, while 16 patients $(39 \%)$ had a stable disease for more than 12 weeks. This type of EMFs provided a safe and well tolerated treatment, as well as evidence of anticancer effects in HCC-patients [95].

\section{Conclusions}

This paper has presented a short review of a new thermodynamic approach to open and complex systems, with particular regards for applications in bioengineering. The basis of this approach is the interaction between the open systems and their environments. The consequence of this interaction is the entropy generation variation which is determined by mass, energy, ions and chemical flows across the boundary of the system. But, the flows represents also the communications between system and environment. It is easy to develop observations of the environment, so the analysis of the entropy generation of the system, but evaluated by the environment can be easily developed.

This approach has been confirmed by the comparisons of its theoretical results and the related experiments in literature, but also by a specific experiment developed in vitro. This approach represents a link between engineering thermodynamics, mechanobiology, biomedical engineering, biology and medicine, with the consequence of becoming a new standpoint in relation to the study of biomedical systems (Figure 1). Consequently, it is the first step to open new frontiers to thermodynamics, the starting point for a new interdisciplinary science for the engineering and physical analysis of biosystems, the bioengineering thermodynamics. If the scientific community of thermodynamics will develop this approach to biosystems, new research frontiers in biomedical and pharmaceutical topics will be open to thermal sciences and engineering.

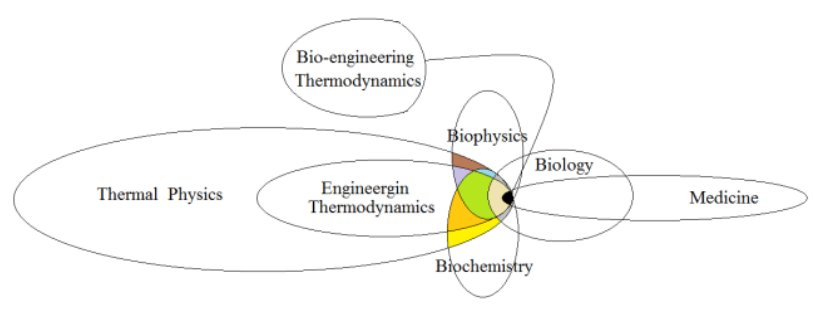

Figure 1. Bioengineering thermodynamics as intersection of the thermodynamics and biological disciplines. 
There exists many methods of cancer treatment, i.e. chemotherapy, radiation therapy, surgery, immunotherapy, monoclonal antibody therapy, etc. The better treatment for the patient is selected by examining the general situation of his health, the type of cancer, the location of tumour, grade of the tumor, and the stage of the disease. Certain kinds of cancer, due to their complexity, require combination of treatments. Often, other unpleasant effects accompany these therapeutic methods, such as fatigue, nausea and vomiting, loss of appetite, pain, hair loss, nerve and muscle effects, metastasis and many others. Consequently, the actual aim of therapeutic research is to increase the therapeutic effect and reduce the unpleasant effects and to allow clinicians to treat certain cancers currently not yet curable [78,79,96]. Electromagnetic fields could be used as a low-cost, safe, and adjuvant treatment of the existing anticancer therapy. There is data supporting the opinion that the use of EMFs has effects on cancer.

Some results in literature confirm our conclusions and the approach itself. But, some consideration must be developed on them. Indeed, there exist some difficulties in the reproducibility of experimental findings, due to not clearly described protocols or not accurate application of them [97100]. Moreover, the exposure of AFC cells did not reveal any significant differences, compared to control, at different EMF intensities and various exposure periods [83] and the static magnetic fields seem not to affect the proliferation of normal cell lines: MRC-5 cells exposed to $3 \mathrm{mT}$ static MF, modulated in amplitude with $3 \mathrm{mT}$ ELF-MF, at $50 \mathrm{~Hz}$, with a superimposition of ELF magnetic field, for $20 \mathrm{~min}$, remained intact and did not show any increase in apoptosis [81]. Furthermore, normal human peripheral blood leukocytes did not undergo DNA fragmentation, when exposed to ELF$\mathrm{EMF}$ at $50 \mathrm{~Hz}, 45 \mathrm{mT}$ ELF-EMF for time periods of 1 and $2.5 \mathrm{~h}$ [82]. But, these fails can be explained as the positive results, by using the thermodynamic approach suggested Indeed, all the theoretical models have been developed to highlight the effect of electromagnetic fields on cells. They use a RC-circuit for the analysis. Some thermodynamic models have been introduces by using local entropy production, but they have the aim to describe the behaviour of the cell in relation to its internal biochemical reaction. Consequently, they have not analysed the different behaviour between normal and cancer cells [101-113].

On the contrary, the thermodynamic approach suggested allows us to describe the effects of the external fields on the whole cell in relation to its metabolism or energy managements, with particular interest to the flows across its membrane, i.e. the interaction between the cell and the environment. Consequently, it allows us to evaluate the frequency and the intensity of the electromagnetic field in relation to each cell geometrical and biophysical properties.

\section{Acknowledgements}

The author must thank Prof. Antonio Ponzetto (Università di Torino) for his scientific support in biomedical interpretation of the thermodynamic results and Prof. Bartolomeo Montrucchio (Politecnico di Torino) for his support in the design of the experimental devices.

\section{Nomenclature}

$\tilde{\mathcal{A}}$ Electrochemical affinity $\left(\mathrm{J} \mathrm{mol}^{-1}\right)$

$\mathcal{A}$ Chemical affinity $\left(\mathrm{J} \mathrm{mol}^{-1}\right)$

c Sound velocity $\left(\mathrm{m} \mathrm{s}^{-1}\right)$

$c_{i}$ Concentration $\left(\mathrm{kg} \mathrm{m}^{-3}\right)$ $d$ Elementary variation; diameter $(\mathrm{m})$

$D$ Diffusion coefficient $\left(\mathrm{m}^{2} \mathrm{~s}^{-1}\right)$

$G$ Mass flow $\left(\mathrm{kg} \mathrm{s}^{-1}\right)$

$h$ Convection coefficient $\left(\mathrm{W} \mathrm{m}^{-1} \mathrm{~K}^{-1}\right)$

$L$ Characteristic length (m)

J General flow $\left(\mathrm{kg} \mathrm{m}^{-2} \mathrm{~s}^{-1}\right)$

$p$ Pressure $(\mathrm{Pa})$

Q Heat flow $\left(\mathrm{W} \mathrm{m}^{-2}\right)$

Pr Prandtl number

$r$ Radius (m)

$R e$ Reynolds number

$s \quad$ Specific entropy $\left(\mathrm{J} \mathrm{kg}^{-1} \mathrm{~K}^{-1}\right)$

$S$ Entropy $\left(\mathrm{J} \mathrm{K}^{-1}\right)$

$t$ Time (s)

$T$ Temperature (K)

$u$ Specific internal energy $\left(\mathrm{J} \mathrm{kg}^{-1}\right)$

$U$ Internal energy $(\mathrm{J})$

$v$ Specific volume $\left(\mathrm{m}^{3} \mathrm{~kg}^{-1}\right)$

$V$ Volume $\left(\mathrm{m}^{3}\right)$

X General force $\left(\mathrm{J} \mathrm{m}^{2} \mathrm{~kg}^{-1}\right)$

$Z \quad$ Specific electric charge $\left(\mathrm{A} \mathrm{s} \mathrm{kg}^{-1}\right)$

$\phi \quad$ Electric potential $\left(\mathrm{W} \mathrm{A}^{-1}\right)$

$\tilde{\mu}$ Electrochemical potential $\left(\mathrm{J} \mathrm{m}^{2} \mathrm{~kg}^{-1}\right)$

$\mu$ Chemical potential $\left(\mathrm{J} \mathrm{m}^{2} \mathrm{~kg}^{-1}\right)$

$\sigma$ Mass density $\left(\mathrm{kg} \mathrm{m}^{-3}\right)$

$\sigma$ Entropy production $\left(\mathrm{W} \mathrm{m}^{-3} \mathrm{~K}^{-1}\right)$

$\tau$ Characteristic time (s)

П Total pressure tensor $(\mathrm{Pa})$

$\nabla \cdot$ Divergence $\left(\mathrm{m}^{-1}\right)$

$\nabla$ Gradient $\left(\mathrm{m}^{-1}\right)$

$\Delta$ Variation

\section{Subscripts}

$B$ Centre of mass

e External interactions; reversible path

$g$ Generation

$i \quad$ For irreversibility; $i$-th element

$m$ Membrane

th Thermal

\section{References}

[1] WHO, World Health Organization, Fact sheet No 297, 2011.Available:www.who.int/mediacentre/factsheets/fs 297/en

[2] I. Verginadis, A. Velalopoulou, I. Karagounis, Y. Simos, D. Peschos, S. Karkabounas and A. Evangelou, Beneficial Effects of Electromagnetic Radiation in Cancer, in S.O. Bashir Ed. Electromagnetic radiation. Shangai: InTech, 2012.

[3] A.E. Pelling, S. Sehati, E.B. Gralla, J.S. Valentineand J.K. Gimzewski, "Local nanomechanical motion of the cell wall of Saccharomyces cerevisiae," Science, 305, 1147-1150, 2004.

[4] J. Wang, D. Lü, D. Mao and M. Long, "Mechanomics: an emerging field between biology and biomechanics," Protein Cells, 5, 518-531, 2014.

[5] C. Bustamante, Y.R. Chemla, N.R. Forde and D. Izhaky, "Mechanical Processes in Biochemistry," Annu. Rev. Biochem., 73, 705-748, 2004. 
[6] S.A. Harris, "Modelling the biomechanical properties of DNA using computer simulation," Phil. Transac. $R$. Soc. A, 364, 3319-3334, 2006.

[7] A. Hudspeth, Y. Choe, A. Mehta and P. Martin, "Putting ion channels to work: mechanoelectrical transduction, adaptation and amplication by hair cells," P.N.A.S., 97, $11765,2000$.

[8] D.F. Wilson, M. Erecińska and P.L. Dutton, "Thermodynamic relationships in mitochondrial oxidative phosphorylation," Annu Rev Biophys Bioeng., 3, 203-230, 1974.

[9] A.R. Waldeck, K. van Dam, J. Berden and P.W. Kuchel, A nonequilibrium thermodynamics model of reconstituted $\mathrm{Ca}^{2+}$-ATPase, Eur. Biophys. J., 27, 255262, 1998.

[10]Q. Jin and C.M. Bethke, "Kinetics of electron transfer through the respiratory chain," Biophys. J., 83, 17971808, 2002.

[11] P. Turina, D. Samurai and P. Graber, "H+/ATP ratio of proton transport-coupled ATP synthesis and hydrolysis catalyzed by CF0F1 \pm liposomes," Eur. Mol. Biol. Org. J., 22, 418-426, 2003.

[12] Y. Demirel, "Modeling of thermodynamically coupled reaction-transport systems," Chem. Eng. J., 139, 106117, 2008.

[13] S. Chowdhury and B. Chanda, "Thermodynamics of electro- mechanical coupling in voltage-gated ion channels," J. Gen. Physiol., 140, 613-623, 2012.

[14] U. Lucia, "Maximum or minimum entropy generation for open systems?," Physica A, 391, 3392-3398, 2012.

[15]S. Dolev and A.C. Elitzur, "Biology and Thermodynamics: Seemingly-Opposite Phenomena in Search of a Unified Paradigm," The Einstein Q.: J. Biol. Med., 15, 24-33, 1998.

[16] U. Lucia, "Quanta and entropy generation," Physica A, 419, 115-121, 2015.

[17] U. Lucia, "Some considerations on molecular machines and Loschmidt paradox," Chem. Phys. Lett., 623, 98$100,2015$.

[18]U. Lucia, "A Link between Nano- and Classical Thermodynamics: Dissipation Analysis (The Entropy Generation Approach in Nano-Thermodynamics)," Entropy, 17, 1309-1328, 2015.

[19] U. Lucia, "Entropy production and generation: clarity from nanosystems considerations," Chem. Phys. Lett., 629, 87-90, 2015.

[20] S. Carnot, Rèflexion sur la puissance motrice du feu sur le machine a dèvelopper cette puissance. Paris: Bachelier Libraire, 1824.

[21]R. Clausius, Mechanical Theory of Heat - with its Applications to the Steam Engine and to Physical Properties of Bodies, London: John van Voorst, 1865.

[22]B.H. Lavenda, Thermodynamics of irreversible processes, Mineola: Dover, 1993.
[23]G. Gouy, "Sur les transformation et l'équilibre en Thermodynamique," C.R. Acad. Sci. Paris, 108, 507509, 1889.

[24] G. Gouy, "Sur l'énergie utilizable," J. Phys., 8, 501-518, 1889.

[25]G. Gouy, "Sur l'énergie utilisable et le potentiel thermodynamique. Note de M. Gouy," C.R. Acad. Sci. Paris, 108, 794, 1889.

[26]P. Duhem, "Sur les transformations et l'équilibre en Thermodynamique. Note de M.P. Duhem," C.R. Acad. Sci. Paris, 108, 666-667, 1889.

[27]A. Stodola, (translated by L.C. Loewenstein), Steam turbine. New York: Van Nostrand, 1905.

[28] U. Lucia, "Mathematical consequences of Gyarmati's principle in rational thermodynamics," Il Nuovo Cimento B, 110, 1227-1235, 1995.

[29]G. Grazzini and U. Lucia, "Global analysis of dissipations due to irreversibility," Rev. Gén. Therm. (Int. J. Therm. Sci.), 36, 605-609, 1997.

[30]U. Lucia, "Irreversibility and entropy in Rational Thermodynamics," Ricerche di Matematica, L1, 77-87, 2001.

[31]U. Lucia, "Probability, ergodicity, irreversibility and dynamical systems," Proc. R. Soc. A-Math., Phys. Eng. Sci., 464, 1089-1104, 2008.

[32]U. Lucia, "Statistical approach of the irreversible entropy variation," Physica A, 387, 3454-3460, 2008.

[33] U. Lucia and G. Gervino, "Hydrodynamic cavitation: from theory towards a new experimental approach", Cent. Europ. J. Phys., 7, 638-644, 2009.

[34] U. Lucia, "Irreversibility, entropy and incomplete information," Physica A, 388, 4025-4033, 2009.

[35]U. Lucia, "Maximum entropy generation and $\kappa$ exponential model," Physica A, 389, 4558-4563, 2010.

[36] U. Lucia, "Irreversible human brain," Med. Hypotheses, 80, 114-116, 2013.

[37]U. Lucia, "Thermodynamics and cancer stationary states," Physica A, 392, 3648-3653, 2013.

[38] U. Lucia, "Thermodynamic paths and stochastic order in open systems," Physica A, 392, 3912-3919, 2013.

[39] U. Lucia, "Entropy generation: From outside to inside!," Chem. Phys. Lett., 583, 209-212, 2013.

[40] U. Lucia, "Entropy generation: Minimum inside and maximum outside," Physica A, 396, 61-65, 2014.

[41] Y.A. Cengel, "Energy, entropy and exergy concepts and their roles in thermal engineering," Entropy, 3, 116-149, 2001.

[42]U. Lucia, "Maximum principle and open systems including two-phase flows," Rev. Gén. Therm. (Int. J. Therm. Sci.), 37, 813-817, 1998.

[43] M. Causà and U. Lucia, "The maximum entropy variation and the calculation of the lattice constant," Il Nuovo Cimento D, 20, 807-810, 1998. 
[44] U. Lucia, "Physical model for the engineering analysis of the thermoelasticity of solid bodies," Chin. J. Mech. Eng., 13, 165-170, 2000.

[45] U. Lucia and G. Maino, "Thermodynamical analysis of the dynamics of tumor interaction with the host immune system," Physica A, 313, 569-577, 2002.

[46] E.G. Gyftopoulos and G.P. Beretta, Thermodynamics. Foundations and Applications. Mineola: Dover Publications, 2005.

[47]H.B. Callen, Thermodynamics and an Introduction to Thermostatistics, Hoboken: John Wiley \& Sons, 1985.

[48] M.W. Zemansky, Heat and Thermodynamics. New York: McGraw-Hill, 1966.

[49]A. Bejan, Advanced Engineering Thermodynamics. Hoboken: John Wiley, 2006.

[50] K.G. Denbigh, "Note on Entropy, Disorder and Disorganization," Brit. J. Phil. Sci., 40, 323-332, 1989

[51] K.G. Denbigh, "The Many Faces of Irreversibility," Brit. J. Phil Sci., 40, 501-518, 1989.

[52] S.G. de Groot and P. Mazur, Non-Equilibrium Thermodynamics, Amsterdam: North-Holland Publishing, 1984.

[53] S.G. de Groot and P. Mazur, Thermodynamics of irreversible processes, Amsterdam: North-Holland Publishing, 1952.

[54] P.W. Atkins, The Elements of Physical Chemistry, 3rd Ed.. Oxford: Oxford University Press, 1992.

[55] U. Lucia, "Irreversibility in biophysical and biochemical engineering," Physica A, 391, 5997-6007, 2012.

[56] U. Lucia and G. Maino, "Entropy generation in biophysical systems," EPL, 101, 56002, 2013.

[57] U. Lucia, "Different chemical reaction times between normal and solid cancer cells," Med. Hypotheses, 81, 58$61,2013$.

[58] U. Lucia, "Molecular refrigerators: a new approach in anti-cancer therapy," OA Med. Hypotheses, 1, 9-12, 2013.

[59]U. Lucia, "Transport processes and irreversible thermodynamics analysis in tumoral systems," Physica A, 410, 380-390, 2014.

[60] J. Serrin, "Conceptual analysis of the Classical Second Law of Thermodynamics," Arch. Rat. Mech. Anal., 70, 355-371, 1979.

[61]D.P. Ruelle, "Extending the definition of entropy to non equilibrium steady states," PNAS, 100, 3054-3058, 2003.

[62] E. Zanchini and G.P. Beretta, "Recent Progress in the Definition of Thermodynamic Entropy," Entropy, 16, 1547-1570, 2014.

[63] G.P. Beretta and E. Zanchini, "Rigorous and General Definition of Thermodynamic Entropy," in Thermodynamics, M. Tadashi (Ed.), Rijeka: InTech, 2011.
[64]T. Volk and O. Pauluis, "It is not the entropy you produce, rather, how you produce it," Phil. Trans. R. Soc. B, 365, 1317-1322, 2010.

[65]U. Lucia, "Stationary open systems: A brief review on contemporary theories on irreversibility," Physica A, 392, 1051-1062, 2013.

[66]U. Lucia, "Transport processes in biological systems: tumoral cells and human brain," Physica A, 393, 327336, 2014.

[67]U. Lucia, "Entropy generation approach to cell systems," Physica A, 406, 1-11, 2014.

[68]U. Lucia, "Entropy generation and cell growth with comments for a thermodynamic anticancer approach," Physica A, 406, 107-118, 2014.

[69] U. Lucia, "Thermodynamic approach to nano-properties of cell membrane," Physica A, 407, 185-191, 2014.

[70]U. Lucia, "Exergy flows as bases of constructal law," Physica A, 392, 6284-6287, 2013.

[71] U. Lucia, "The Gouy-Stodola Theorem in Bioenergetic Analysis of Living Systems (Irreversibility in Bioenergetics of Living Systems)," Energies, 7, 57175739, 2014.

[72]U. Lucia, A. Ponzetto and T.S Deisboeck, "A thermophysical analysis of the proton pump vacuolar-ATPase: the constructal approach," Sci. Rep., 4, 6763, 2014.

[73]U. Lucia, A. Ponzetto and T.S. Deisboeck, "A thermodynamic approach to the 'mitosis/apoptosis' ratio in cancer," Physica A, 436, 246-255, 2015.

[74]U. Lucia, G. Grazzini, B. Montrucchio, G. Grisolia, R. Borchiellini, G. Gervino, C. Castagnoli, A. Ponzetto and F. Silvagno, "Constructal thermodynamics combined with infrared experiments to evaluate temperature differences in cells," Scie. Rep., 5, 11587, 2015.

[75] Y.C. Chen, C.C. Chen, W. Tu, Y.T. Cheng and F.G. Tseng, "Design and fabrication of a microplatform for the proximity effect study of localized ELF-EMF on the growth of in vitro HeLa and PC-12 cells," J. Micromech. Microeng, 20, 125023, 2010.

[76] S. Tuffet, R. de Seze, J.M. Moreau and B. Veyret, "Effects of a strong pulsed magnetic field on the proliferation of tumor cells in vitro," Bioelectrochem. Bioener., 30, 151-160, 1993.

[77] C. Morabito, S. Guarnieri, G. Fanò and M.A. Mariggiò, "Effects of acute and chronic low frequency electromagnetic field exposure on PC12 cells during neuronal differentiation," Cell Physiol Biochem, 24, 947-958, 2010.

[78] M.J. Ruiz Gómez, J.M. Pastor Vega, L. de la Peña, L. Gil Carmona, and M. Martínez Morillo, "Growth modification of human colon adenocarcinoma cells exposed to a low-frequency electromagnetic field," $J$. Physiol. Biochem., 55, 79-83, 1999.

[79]M.J. Ruiz Gómez, L. de la Peña, J.M. Pastor, M. Martínez Morillo and L. Gil, "25 Hz electromagnetic field exposure has no effect on cell cycle distribution and apoptosis in U-937 and HCA-2/1cch cells," Bioelectrochemistry, 53, 137-140, 2000. 
[80] R.R. Raylman, A.C. Clavo and R.L. Wahl, "Exposure to strong static magnetic field slows the growth of human cancer cells in vitro," Bioelectromagnetics, 17, 358-363, 1996.

[81] S. Tofani, D. Barone, M. Cintorino, M.M. de Santi, A. Ferrara, R. Orlassino, P. Ossola, F. Peroglio, K. Rolfo and F. Ronchetto, "Static and elf magnetic fields induce tumour growth inhibition and apoptosis," Bioelectromagnetics, 22, 419-428, 2001.

[82] T. Hisamitsu, K. Narita, T. Kashara, A. Seto, Y. Yu and K. Asano, "Induction of apoptosis in human leukemic cells by magnetic fields," Jpn. J. Physiol., 47, 307-310, 1997.

[83] M. Simkó, R. Kriehuber, D.G. Weiss and R.A. Luben, "Effects of $50 \mathrm{~Hz}$ EMF exposure on micronucleus formation and apoptosis in transformed and nontransformed human cell lines," Bioelectromagnetics, 19, 85-91, 1998.

[84] M. Buttiglione, L. Roca, E. Montemurno, F. Vitiello, V. Capozzi and G. Cibell, "Radiofrequency radiation (900 $\mathrm{MHz}$ ) induces egr-1 gene expression and affects cellcycle control in human neuroblastoma cells," J. Cell. Physiol., 213, 759-767, 2007.

[85] S. Yamaguchi, M. Ogiue-Ikeda, M. Sekino and S. Ueno, "Effects of pulsed magnetic stimulation on tumour development and immune functions in mice," Bioelectromagnetics, 27, 64-72, 2006.

[86] R. de Seze, S. Tuffet, J.M. Moreau and B. Veyret, "Effects of $100 \mathrm{mT}$ time varying magnetic fields on the growth of tumours in mice," Bioelectromagnetics, 21, 107-111, 2000.

[87] C.D. Williams, M.S. Markov, W.E. Hardman and I.L. Cameron, "Therapeutic electromagnetic field effects on angiogenesis and tumour growth," Anticancer Research, 21, 3887-3892, 2001.

[88] X. Zhang, H. Zhang, C. Zheng, C. Li, X. Zhang and W. Xiong, "Extremely low frequency (ELF) pulsedgradient magnetic fields inhibit malignant tumour growth at different biological levels" Cell Biol. Inter., 26, 599-603, 2002.

[89] M.N. Jimenez-Garcia, J. Arellanes-Robledo, D.I. Aparicio Bautista, M.A. Rodriguez-Segura, S. VillaTrevino and J.J. Godina-Nava, "Anti-proliferative effect of an extremely low frequency electromagnetic field on preneoplastic lesions formation in the rat liver," B.M.C. Cancer, 24, 159, 2010.

[90] S. Tofani, M. Cintorino, D. Barone, M. Berardelli, M.M. De Santi, A. Ferrara, R. Orlassino, P. Ossola, K. Rolfo, F. Ronchetto, S.A. Tripodi and P. Tosi, "Increased mouse survival, tumour growth, inhibition and decreased immunoreactive p53 after exposure to magnetic fields," Bioelectromagnetics, 23, 230-238, 2002.

[91] A. Barbault, F.P. Costa, B. Bottger, R.F. Munden, F. Bomholt, N. Kuster and B. Pasche, "Amplitudemodulated electromagnetic fields for the treatment of cancer: discovery of tumour-specific frequencies and assessment of a novel therapeutic approach," J. Exp. Clin. Cancer Res., 28, 51, 2009.
[92]F. Ronchetto, D. Barone, M. Cintorino, M. Berardelli, S. Lissolo, R. Orlassino, P. Ossola and S. Tofani, "Extremely low frequency-modulated static magnetic fields to treat cancer: A pilot study on patients with advanced neoplasm to assess safety and acute toxicity," Bioelectromagnetics, 25, 563-571, 2004.

[93]E.D. Kirson, V. Dbaly, F. Tovarys, J. Vymazal, J.F. Soustiel, A. Itzhaki, D. Mordechovich, S. SteinbergShapira, Z. Gurvich, R. Schneiderman, Y. Wasserman, M. Salzberg, B. Ryffel, D. Goldsher, E. Dekel and Y. Palti, "Alternating electric fields arrest cell proliferation in animal tumour models and human brain tumour," PNAS, 104, 10152-10157, 2007.

[94] M. Salzberg, E. Kirson, Y. Palti and C. Rochlitz, "A pilot study with very lowintensity, intermediatefrequency electric fields in patients with locally advanced and/or metastatic solid tumours," Onkologie, 31, 362-365, 2008.

[95]F.P. Costa, A.C. de Oliveira, R. Meirelles, M.C. Machado, T. Zanesco, R. Surjan, M.C. Chammas, M. de Souza Rocha, D. Morgan, A. Cantor, J. Zimmerman, I. Brezovich, N. Kuster, A. Barbault and B. Pasche, "Treatment of advanced hepatocellular carcinoma with very low levels of amplitude-modulated electromagnetic fields," $\mathrm{Br}$. J. Cancer, 105, 640-648, 2011.

[96] J.R. Gray, C.H. Frith and J.D. Parker, "In vivo enhancement of chemotherapy with static electric or magnetic fields," Bioelectromagnetics, 21, 575-583, 2000 .

[97] A. Lacy-Hulbert, R.C. Wilkins, T.R. Hesketh and J.C. Metcalfe, "No effect of $60 \mathrm{~Hz}$ electromagnetic fields on MYC or beta-actin expression in human leukemic cells," Radiat Res, 144, 9-17, 1995.

[98] R.S. Malyapa, C. Bi, E.W. Ahern, and J.L. Roti, "Detection of DNA damage by the alkaline comet assay after exposure to low-dose gamma radiation," Radiat Res, 149, 396-400, 1998.

[99] J.D. Saffer and S.J. Thurston, "Short exposures to $60 \mathrm{~Hz}$ magnetic fields do not alter MYC expression in HL60 or Daudi cells," Radiat Res, 144, 18-25, 1995.

[100] M. Jin, H. Lin, L. Han, M. Opler, S. Maurer, M. Blank and R. Goodman, "Biological and technical variables in myc expression in HL60 cells exposed to $60 \mathrm{~Hz}$ electromagnetic fields," Bioelectrochem. Bioenerg, 44, 111-120, 1997.

[101] D.J. Panagopoulos, Electromagnetic interaction between environmental fields and living systems determines heath and well being, in M.H. Kwang and S.O. Yoon, Electromagnetic Fields: Principles, Engineering Applications and Biophysical Effects. Hauppauge (USA): Nova Science Publisher, 2013.

[102] A. Bejan and Y. Ventikos, "Celebral oxygenation and optimal vascular brain orgamization," J. R. Soc. Interface, 12, 20150245, 2015.

[103] E.D. Kirson, Z. Gurvich, R. Schneiderman, E. Dekel, A. Itzhaki, Y. Wasserman, R. Schatzberger, and Y. Palti, "Disruption of cancer cell replication by 
alternating electric fields," Cancer Research, 64, 3288-3295, 2004.

[104] D. Kondepudi and I. Prigogine, Modern Thermodynamics, From Heat Engine to Dissipative Structures, New York: Wiley, 1999.

[105] F.L Luo, "Entropy production in cell and reversal of entropy flow as an anticancer therapy," Front. Phys. China, 4, 122-136. 2009

[106] L.F. Luo, J. Molnar, H. Ding, X. Lv, and G. Spengler, "Physicochemical attack against solid tumors based on the reversal thermodynamics in anticancer therapy," Diagn Pathol, I, 43-49, 2006.

[107] L.F. Luo, J. Molnar, D. Hui, X. Lv and G. Spengler, "Attempts to introduce thermodynamics in anticancer therapy using entropy production difference between cancer and normal cells," Acta Scientiarum Naturalium Univ NeiMongol, 37, 295-303, 2006.

[108] T. Mäkelä and A. Annila, "Natural patterns of energy dispersal," Phys. Life Rev., 7, 477-498, 2010.

[109] W.B. Mercer, (2014, October, 8th,). The living Cell as an Open Thermodynamic System: Bacteria and
Irreversible Thermodynamics, No. SMUFDTECHNICAL MANUSCRIPT-640, Fort Detrick: Frederick, MD, USA [Online]. Available: http://www.dtic.mil/dtic/tr/fulltext/u2/726932.pdf.

[110] G. Nicolis, Stability and Dissipative Structures in Open Systems Far from Equilibrium, in I. Progogine and S.A. Rice (Eds.), Advances in Chemical Physics. New York: Wiley-Interscience, 1971.

[111] J. Pokorný, J. Hašek and F. Jelínek, "Endogenous Electric Field and Organization of Living Matter," Electromagn. Biol. Med., 24, 185-197, 2005.

[112] I. Prigogine, Structure, dissipation and life, in $M$. Marois Ed., Theoretical Physics and Biology. NorthHolland: Amsterdam, 1969.

[113] I. Prigogine, "Time, structure, and fluctuations," Science, 201, 777-785, 2001. 